\title{
Thermal Measurements in Conductive Heat Transfer Tree-Like Structures Obtained by Topology Optimization
}

\author{
Vignaesh Subramaniam $^{1,2}$, Talib Dbouk ${ }^{1,2}$, Jean-Luc Harion ${ }^{1,2}$ \\ ${ }^{1}$ IMT Lille Douai, Industrial Energy Department \\ F-59500 Douai, France \\ ${ }^{2}$ University of Lille \\ F-59000 Lille, France \\ vignaesh.subramaniam@imt-lille-douai.fr; talib.dbouk@imt-lille-douai.fr; jean-luc.harion@imt-lille-douai.fr
}

\begin{abstract}
Topology optimization (TO) applied to structural design has undergone a tremendous development since its introduction by Bendsøe and Kikuchi in 1988. However, topology optimization applied to heat transfer and fluid flows is still not mature as compared to its structural counterpart. Moreover, to date and to author's knowledge, no experimental study on thermal optimized configurations exists in the literature. Therefore, the main objective of this study is focused on experimental examination of conductive heat transfer tree-like structures obtained numerically by topology optimization (TO). In the configuration presented in this communication, TO is implemented to define thermally optimal solutions for cooling heat volumes generating by organizing a high conductivity metallic material as complex tree-like structure inside regions of lower thermal conductivity heat generating material. Experimental thermal measurements are carried out on the optimal structure using infrared thermography in order to qualify the heat transfer performances of the geometries obtained by the developed topology optimization numerical method.
\end{abstract}

Keywords: Topology Optimization, Heat Conduction, Volume-To-Point Problem, Infrared Thermography.

\section{Nomenclature}

A Length of the domain, $m$

$C \quad$ Size of the heat sink, $m$

$C_{p} \quad$ Specific heat capacity, $\mathrm{J} \mathrm{kg}^{-1} \mathrm{~K}^{-1}$

$D \quad$ Height of thermal insulation, $\mathrm{mm}$

$e$ Thickness, $\mathrm{mm}$

$f_{0} \quad$ Objective function

$k \quad$ Thermal conductivity, $W \cdot m^{-1} K^{-1}$

$h_{\text {ext }} \quad$ Convective heat transfer coefficient, $W \cdot m^{2} K^{1}$

$L_{x} \quad$ Maximum dimension in $x$-direction, $m$

$L_{x} \quad$ Maximum dimension in $y$-direction, $m$

n normal vector

$M^{2} \quad$ Number of square elements

OS Object signal, digital level $(D L)$

$p \quad$ Penalization factor

$\dot{q} \quad$ Volumetric heat generation rate, $W \cdot m^{-3}$

$T$ Temperature, $K$

$\begin{array}{ll}\tilde{T} & \text { Normalized temperature } \\ \tilde{x}, \tilde{y} & \text { Normalized system of coordinates } \\ \boldsymbol{x}_{j} & \text { Design variable vector } \\ \text { Greek } & \text { symbols } \\ \alpha & \text { Thermal diffusivity, } \mathrm{m}^{2} . \mathrm{s}^{-1} \\ \gamma & \text { Ratio of thermal diffusivity } \\ \Delta x, \Delta y & \text { Mesh size, } \mathrm{mm} \\ \varepsilon & \text { Convergence criterion } \\ \rho & \text { Density, }{\mathrm{kg} . \mathrm{m}^{-3}}^{-3} \\ \varphi_{\text {max }} & \text { Maximum volume fraction, } \% \\ \Omega & \text { Dimensions of the optimal structure, } \mathrm{mm} \\ \text { Indices and exponents } \\ 0 & \text { Material of lower thermal diffusivity } \\ 1 & \text { Material of higher thermal diffusivity } \\ \text { eff } & \text { Effective }\end{array}$

\section{Introduction}

Designing efficient thermal systems is a very crucial step for developing new products. In the automotive industry, next-generation engines, motors, generators and converters are designed more compactly for reducing mass, minimizing required space, and increasing fuel efficiency. This compactness makes power density of equipment higher and causes thermal load to constantly increase. As a consequence, the ability to efficiently remove heat from an increasingly restricted space is currently a very critical issue. The need to develop optimization methodologies in order to design 
efficient cooling systems is currently drawing the attention of a large number of industrial and university researchers. Sizing, shape and topology optimization methods have great potential to advance cooling structure design. The rapid growth of computational power and Computer-Aided-Engineering (CAE) software makes it possible to model complex geometries and accurately take into account physical processes and material behaviour with reasonable computational efforts.

Of the automated optimization methods that exist in literature, namely topology, size and shape, topology optimization (TO) is the most recent one which was initially introduced by Bendsøe and Kikuchi [1]. Recently, it has been successfully applied to wide range of applications including thermal system when an optimal material distribution is required. One such motivation is to minimizing the thermal resistance within a small volume commonly referred as the volume-to-point (VP) heat conduction problem [2-4]. Although, many numerical studies have been conducted for topology optimization applied to VP heat conduction problem, to author's knowledge, no experimental investigation of topology optimized configuration exists in literature which questions the validity of $\mathrm{TO}$ algorithms for producing optimized solutions for real world applications.

Therefore, the objective of this study is to analyse experimentally the results obtained from a topology optimization method applied to a volume-to-point heat conduction problem. This will question the validity of developed topology optimization numerical tools in really producing efficient cooling/heating industrial devices.

\section{Experimental Examination of the Topology Optimized Configuration}

\subsection{Problem Definition}

Topology optimization constitutes the distribution of elemental sized material, within a domain, in such a manner to optimize (i.e. minimize/maximize) a specified performance index. In the current scenario, the performance index is the overall average temperature in the domain. However, other functions may also be used to describe the thermal performance of the system, for example the maximum temperature of the domain $T_{\max }$ as proposed by Gersborg-Hansen et al. [2] or the multi-objective cost function proposed by Marck et al. [5] (minimization of the mean temperature and the temperature difference on the domain).

Fig. 1 represents the 2-D computational domain of a heat conduction topology optimization problem. The domain has lengths of $A(m)$ in the both the $x$ and $y$ directions and the thickness $(e)$ is considered negligible as compared to the side $A$ $(e \ll A)$. All the boundaries are adiabatic except for the small centre part of the lower boundary (length $C$ ) which is at a constant temperature of $T_{0}$ and thus acts as a heat sink. Heat flow may only escape from the heat sink at the lower boundary. An effective constant volumetric heat generation rate $\dot{q}_{\text {eff }}\left[\mathrm{W} \mathrm{m}^{-3}\right]$ is applied as heat source. Under these conditions, the aim is to find the best topology of material of thermal diffusivity $\alpha_{1}$ with an objective to minimize the overall average temperature in the domain.
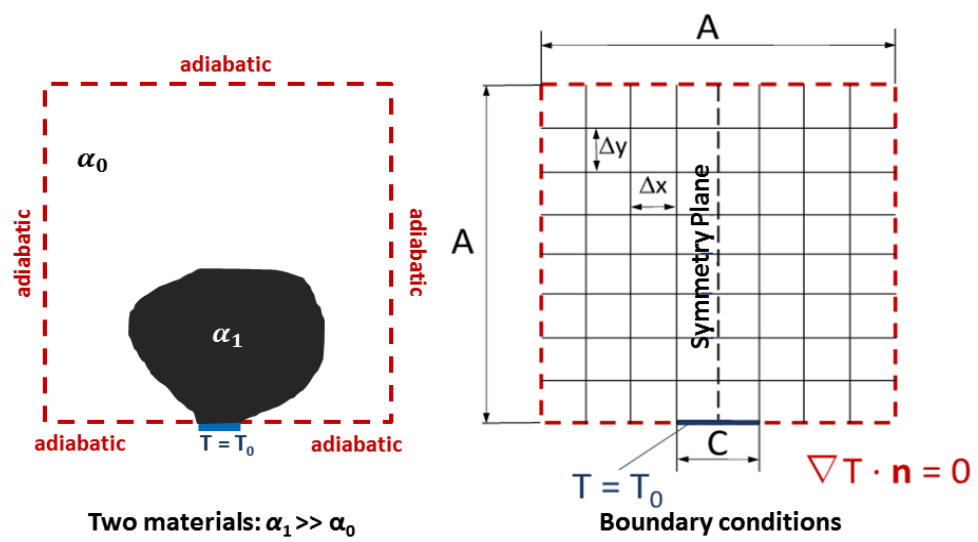

Fig. 1: 2D heat conduction topology optimization problem and associated boundary conditions.

Mathematically, the above TO problem can be expressed as follows: 


$$
\left\{\begin{array}{c}
\text { Minimize: } f_{0}=\frac{1}{M^{2}} \sum_{j=1}^{M^{2}} T_{j} \\
\text { Subject to: } \\
\nabla \cdot(1 \mathrm{a}) \\
\frac{1}{\left.\alpha_{e f f} \nabla T\right)+S_{e f f}(x, y)=0} \quad \text { (1b) } \sum_{j=1}^{M^{2}} x_{j} \leq \varphi_{\max } \quad(1 \mathrm{c})
\end{array}\right\}
$$

Where $f_{0}$ is the objective function to be minimized, subjected to a steady state heat equation $1(\mathrm{~b})$ and an inequality constraint 1(c). The amount of high thermal conductive material is limited by restricting the ratio of its effective volume to the total domain volume, given by $\varphi_{\max }$ i.e. the maximum allowed relative quantity of material of high thermal diffusivity. $M^{2}$ is the number of square elements used to discretize uniformly the 2D domain in a Cartesian frame of reference. $\alpha_{e f f}$ is the effective thermal diffusivity in $\left[\mathrm{m}^{2} \mathrm{~s}^{-1}\right] . S_{\text {eff }}$ is a thermal source term such that:

$$
S_{e f f}=\frac{\dot{q}_{e f f}}{\rho_{e f f} C_{p_{e f f}}}
$$

$T$ is the temperature in Kelvin $[K]$ and $x_{j}=\left(x_{1}, x_{2}, \ldots, x_{M^{2}}\right)$ represents the design variable vector describing two materials such that $x=1$ for material with diffusivity $\alpha_{1}$ and $x=0$ for material with diffusivity $\alpha_{0}$.

In such topology optimization problems, the optimal solution is represented by integer discrete variables $x_{j}(0-1$ values) which show clearly the optimal distribution of two materials in the domain. However, it is numerically very difficult to reach direct solutions using directly defined discrete variables formulation. To avoid this difficulty, the Solid Isotropic Material with Penalization (SIMP) technique [6] is used in the literature. The SIMP technique replaces the discrete integer variables with continuous ones, and then introduces some kind of penalty that steers the solution towards discrete $0-1$ values as the following:

$$
\begin{gathered}
S_{e f f}=S_{0}+x_{j}^{p}\left(S_{1}-S_{0}\right) \\
\alpha_{e f f}=\alpha_{0}+x_{j}^{p}\left(\alpha_{1}-\alpha_{0}\right)
\end{gathered}
$$

With $p \geq 1$ and the subscript indices 0 and 1 represents two materials of different thermal conductivities. For the current problem, $x_{j}(t=0)=\eta_{\text {initial }} \forall j$ (initially homogeneous material); $\gamma=\alpha_{1} / \alpha_{0}=760 ;$ and $\varphi_{\max }=30 \%$. Density filtering technique introduced by Bruns and Tortorelli [7] is used with a filter radius of $1.428 \mathrm{~mm}$. A stopping convergence criterion is defined based on the design variable steadiness during the latest iterations (when the squarednorm of the Karush-Kuhn-Tucker (KKT) conditions becomes less than a positive real number $\varepsilon$ such that $\varepsilon \ll 1$ ).

\subsection{Numerical Methods}

The system of equations (1) is solved by using the method of moving asymptotes (MMA) algorithm introduced by Svanberg [8]. The method of moving asymptotes algorithm is a gradient based optimization algorithm which is used to solve a non-linear optimization problem by introducing a strictly convex sub-problem. Recently, Dbouk and Harion [9] investigated in detail the performance of MMA algorithm for optimization problems. A continuous adjoint system is used in this study to feed the MMA algorithm [10].

The SIMP material interpolation technique is used in order to achieve design variables of discrete nature for a twomaterial representation (values 0 or 1). The partial differential equation 1(b) is discretized spatially over the computational domain of Fig.1 using the Finite Volume Method (FVM) in order to find the temperature field (using a structured uniform square-cells mesh of $\Delta x=\Delta y$ with $M=140$ ). 


\subsection{Objective of the Experiment}

The TO method provides a "tree-like" material distribution of the high diffusivity material for heat evacuation from the areas of low diffusivity material as shown in Fig. 2. On the left the repartition values of high and low diffusivities materials are shown and the right hand side presents the final optimized configurations obtained.
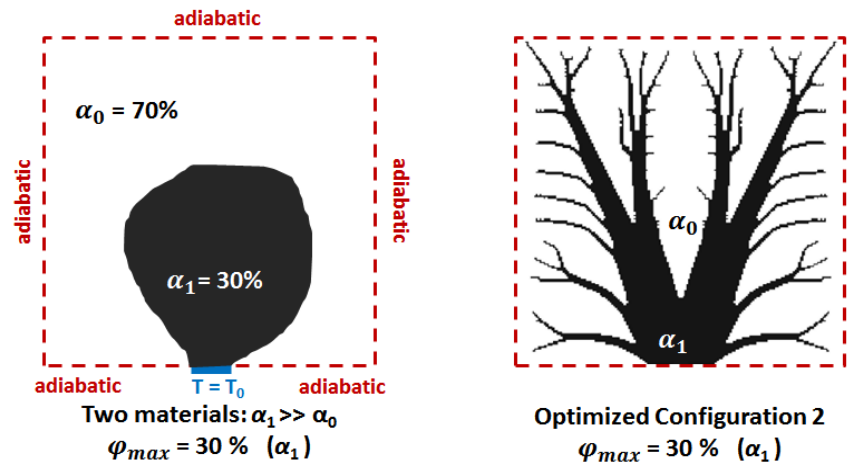

Fig. 2: Topology optimized configuration for VP heat conduction problem.

The objective of the current experiment is to analyse the thermal performance of the above optimal structure under some specific thermal loads and boundary conditions. For the current scenario, the thermal performance criteria is the overall average temperature in the domain which was also the objective function for the TO numerical process.

\subsection{Experimental Setup}

The heat transfer problem in this experiment is analyzed under steady-state conditions. Some important requirements of the experiment which characterizes the test section are as follows:

1) The optimal structure is fabricated for the dimensions $\Omega=100 \mathrm{~mm} \times 100 \mathrm{~mm}$.

2) The heat sink size $(\mathrm{C})$ corresponds to the relation $\mathrm{C} / \mathrm{A}=0.05$.

3) The high diffusivity material should respect the volume constraint of $\varphi_{\max }=30 \%$.

4) The thermal diffusivity of the materials should be in the ratio $\gamma=\alpha_{1} / \alpha_{0}=760$.

One of the main critical aspects in performing this experiment is the proper selection of the two materials. Fundamentally being a heat transfer problem, the selected materials should allow for expansion or contraction during heating and cooling, without separating from each other at least under operating temperature range. After testing many combinations for the two materials, aluminum and a specific polymer were selected as the high diffusivity and low diffusivity material, respectively. The thermal properties of the two materials are listed in Table 1.

Table 1: Material properties for Aluminum and polymer.

\begin{tabular}{|c|l|l|l|l|}
\hline \multirow{2}{*}{ Material } & $\boldsymbol{k}$ & $\boldsymbol{\rho}$ & $\boldsymbol{C}_{\boldsymbol{p}}$ & $\boldsymbol{\alpha}$ \\
\cline { 2 - 5 } & $\mathrm{W} \cdot \mathrm{m}^{-1} \mathrm{~K}^{-1}$ & $\mathrm{~kg} \cdot \mathrm{m}^{-3}$ & $\mathrm{~J} \cdot \mathrm{kg}^{-1} \mathrm{~K}^{-1}$ & $\mathrm{~m}^{2} \cdot \mathrm{s}^{-1}$ \\
\hline Aluminum & 214.04 & 2700 & 891 & $8.89 \times 10^{-5}$ \\
\hline Polymer & 0.19663 & 1100 & 1522.72 & $1.17 \times 10^{-7}$ \\
\hline
\end{tabular}

The next step is to fabricate the test section with the two selected materials in such a way that it represents exactly the material distribution obtained from the TO solver (see Fig. 2). This fabrication is a two-step process where in the first step the aluminum sheet of a prescribed thickness is cut in the shape of the tree structure using water-jet cutting and in the second step the polymer is molded around the tree structure to fill the design domain of $\Omega=100 \mathrm{~mm} \times 100 \mathrm{~mm}$.

The TO problem in section 2.1 dealt with heat evacuation from the low conductivity heat generating volumes. A heat generating volume generates heat from within the volume, and can be approximated by applying a constant uniform heat flux on the surface of the test section if provided the test section is very thin. A classical silicone rubber thin film heater 
was selected as the surface heat source $\left(W^{-2}\right)$ applied in this experiment. To extract heat from the system, a constant lower wall temperature is required at the base of the tree (here $0^{\circ} \mathrm{C}$ ). A special high conductivity (copper) cooling plate with an inner water circulation that is accurately thermo-regulated $\left( \pm 0.01{ }^{\circ} \mathrm{C}\right.$ of stability) is used for this purpose. In addition, a thermal paste is also applied between the surfaces to further enhance the heat transfer capability.

The thickness $(e)$ is chosen equal to $1.5 \mathrm{~mm}$ after preliminary 3D CFD confirmation that the transverse temperature gradient (through the thickness of the structure) is negligible for this thickness. The steady state temperature distribution on the test section is measured using infrared (IR) thermography. High-emissivity black paint is uniformly applied over the entire measurement surface to enhance IR acquisition. The IR camera was calibrated before using it in each measurement. The final experimental set-up is sketched in Fig. 3. The experimental test bench is the integration of test section and the various elements required to perform the experiment.

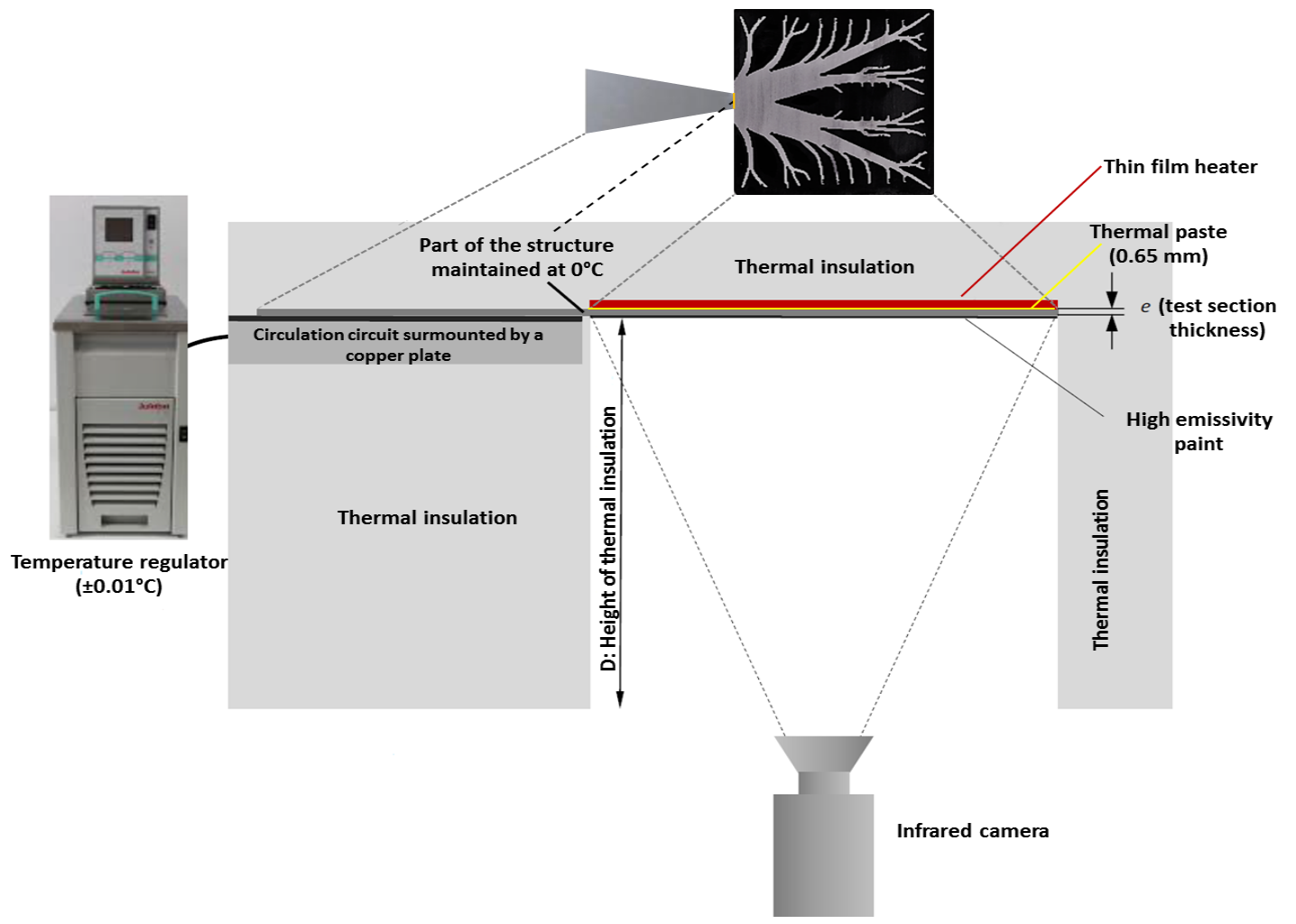

Fig. 3: Schematic representation of the experimental test bench.

\section{Results}

For performing IR measurement, the thin film heater provides a surface heat flux of $588 \mathrm{~W} \mathrm{~m}^{-2}$ over the surface of the test section. The heat sink temperature is maintained at $0{ }^{\circ} \mathrm{C}$ at the isothermal boundary (see Fig. 1: $T=T_{0}$ ). All other boundaries are well insulated except for the bottom face. The steady-state is achieved when the temperature values in the domain stops changing and then the IR measurements are taken on the bottom face. The raw images from the IR camera are then post-processed in Matlab ${ }^{\circledR}$ to better interpret the results.

The results obtained from IR thermal acquisitions cannot be compared directly to those obtained from the TO solver. Instead, the optimal structure obtained from TO (refer Fig. 2) is simulated into a 3D CFD conjugate heat transfer numerical solver to mimic exactly the same initial and boundary conditions that exists during the experiments. In this way, one can efficiently investigate the thermal performance of the topology optimized structure by drawing a direct comparison between the CFD numerical results and IR thermal measurements. 3D CFD simulations are performed in STAR-CCM+ for the optimal structure to obtain steady state temperature profile. To better compare the results obtained from the two sources, the temperature and the lengths are normalized as follows: 


$$
\begin{gathered}
\tilde{T}=\frac{T-T_{\min }}{T_{\max }-T_{\min }} \\
\tilde{x}=\frac{x}{L_{x}} \\
\tilde{y}=\frac{y}{L_{y}}
\end{gathered}
$$

where $\widetilde{T}$ is the normalized temperature, $T_{\min }$ is the minimum temperature in the structure (isothermal boundary at the heat sink $\left.T_{0}\right), T_{\max }$ is the highest temperature observed in the structure for each measurement and $T$ is the actual temperature in ${ }^{\circ} \mathrm{C}$ at each pixel (IR measurement) or cell (CFD simulation). Similarly, $\tilde{x}$ and $\tilde{y}$ are the normalized $x$ and $y$ coordinates, respectively, and, $L_{x}$ and $L_{y}$ are the maximum dimension in $x$ and $y$-directions, respectively.

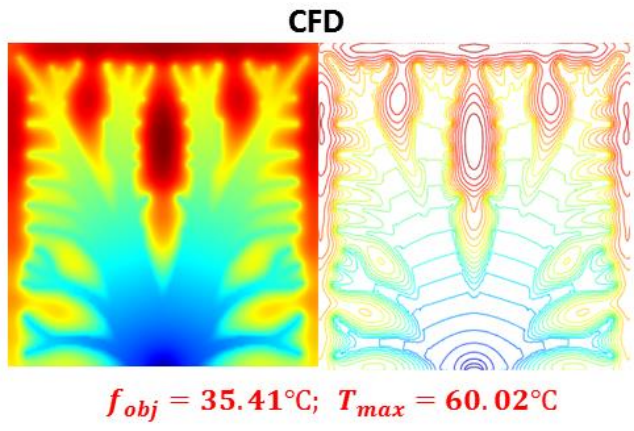

(a) Normalized temperature contours and isotherms

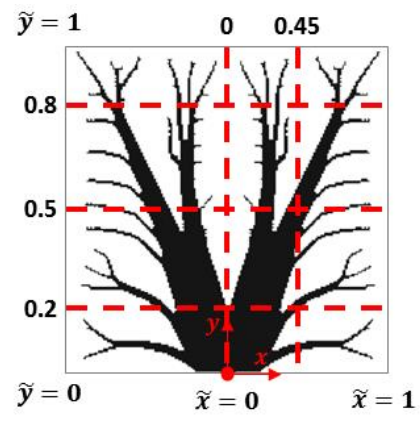

(b) TO structure $\left(\varphi_{\max }=28.6 \%\right)$

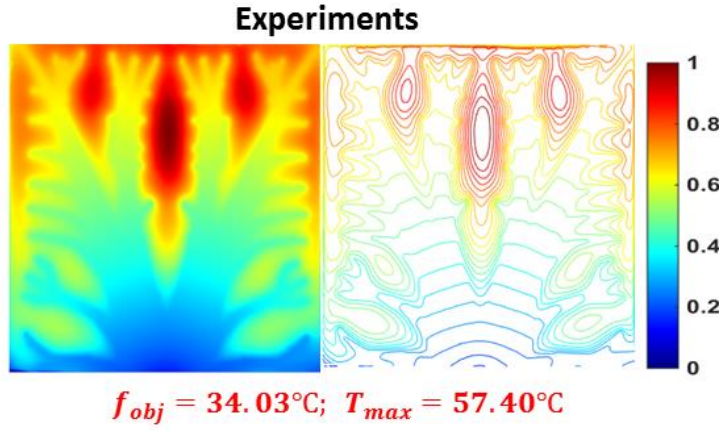

(c) Normalized temperature contours and isotherms

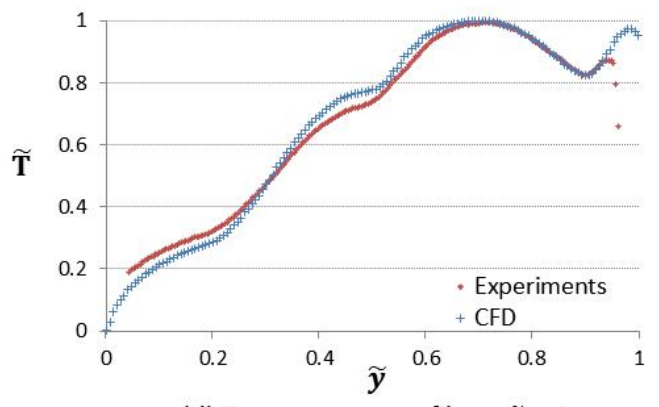

(d) Temperature profile at $\tilde{x}=0$

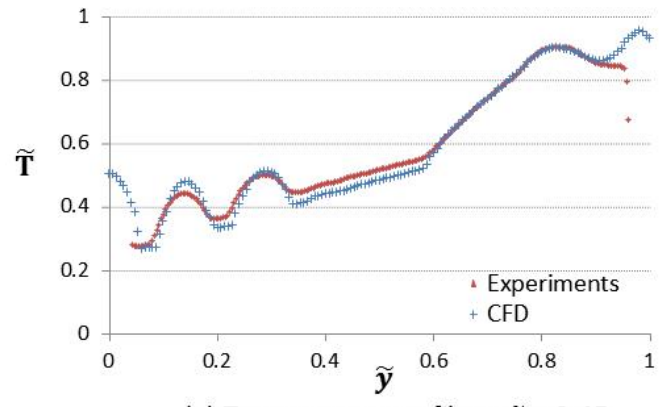

(e) Temperature profile at $\tilde{x}=0.45$

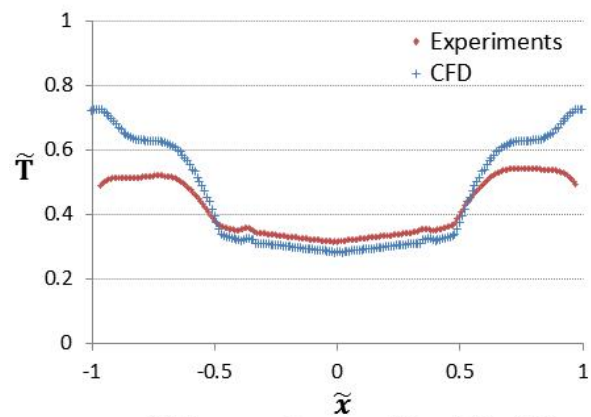

(f) Temperature profile at $\tilde{y}=0.2$

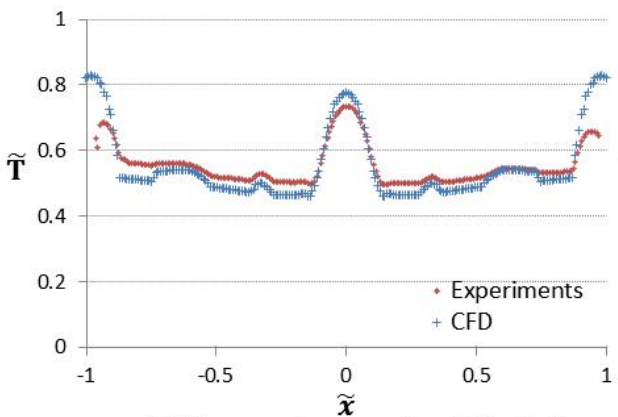

(g) Temperature profile at $\tilde{y}=0.5$

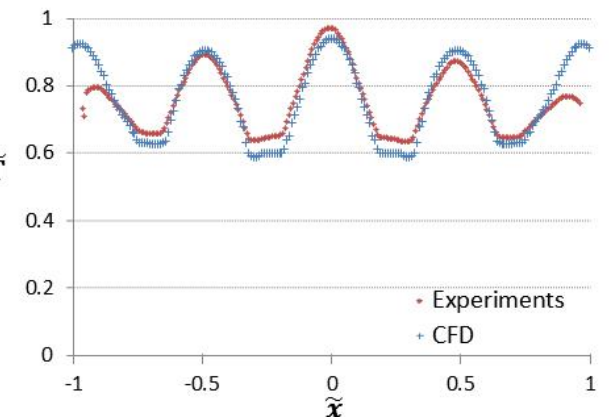

(h) Temperature profile at $\tilde{y}=0.8$

Fig. 4: Normalized steady state temperature measurement for the topology optimized structure with $\varphi_{\max }=30 \%$. 
For the optimal structure with $\varphi_{\max }=30 \%$, Fig. 4(a) shows the normalized steady state temperature field and isotherms as obtained by CFD simulations, whereas Fig. 4(c) demonstrates the results obtained by IR thermal measurements. In the IR measurements, an average temperature $\left(f_{o b j}\right)$ of $34.03^{\circ} \mathrm{C}$ and a maximum temperature $\left(T_{\max }\right)$ of $57.40^{\circ} \mathrm{C}$ are observed in the domain. On the other hand, CFD simulations provide an average temperature and maximum temperature of $35.41{ }^{\circ} \mathrm{C}$ and $60.02^{\circ} \mathrm{C}$, respectively.

The accuracy and high spatial resolution of the IR thermography method facilitates clear visualization of heat transfer from individual branches of the tree structure (see Fig. 4(c)). As a consequence, the value of objective function and maximum temperature measured by IR thermography and to those obtained by CFD simulations are very close.

To further investigate the comparison between the results obtained by IR measurements and CFD, the temperature profiles are extracted and compared at five different locations in the domain (see Fig. 4(b)), the process being termed here as "line probe measurements". These line probe measurements are performed at three horizontal and two vertical lines in the domain and compared with the CFD data. Figure 4(d) and 4(e) show the temperature profiles at vertical lines $\tilde{x}=0$ and $\widetilde{x}=0.45$, respectively. Figure $4(\mathrm{f}), 4(\mathrm{~g})$ and $4(\mathrm{~h})$ show the temperature profiles at horizontal lines $\tilde{y}=0.2, \tilde{y}=0.5$ and $\tilde{y}=0.8$, respectively. The temperature profiles measured at these locations clearly demonstrate the closeness of the results obtained from CFD and IR measurements. Moreover, when examined closely, one can clearly observe the accuracy with which the IR thermography in the current experimental set-up captures even the smallest of the temperature changes in the system.

Another important observation that can be made in Fig. 4 from the temperature contours is the significant temperature drop that occurs near the edges of the domain for the experimental results. Although important precautions are made to ensure good insulation at edges in the experimental set-up, it is very difficult to reproduce a near-perfect insulating boundaries like those modelled in the CFD simulations. Hence, the small differences that exist between the two results can be attributed to the conditions prevailing at the boundary, in other words the heat loss from the boundary.

To test the reproducibility of results, same experiments are performed again on additional sample fabricated for the given optimal structure. Table 2 summarises the maximum temperature, average temperature and standard deviation for the two measurements.

Table 2: Reproducibility of infrared measurements for the topology optimized structure with $\varphi_{\max }=30 \%$.

\begin{tabular}{|c|c|c|c|}
\hline S. No. & $\boldsymbol{T}_{\max }\left({ }^{\circ} \boldsymbol{C}\right)$ & $\boldsymbol{T}_{\boldsymbol{a v g}}\left({ }^{\circ} \boldsymbol{C}\right)$ & $\boldsymbol{\sigma}$ \\
\hline $1^{\text {st }}$ measurement & 57.40 & 34.03 & 9.33 \\
\hline $2^{\text {nd }}$ measurement & 56.95 & 33.21 & 9.85 \\
\hline
\end{tabular}

It can be seen from Table 2 that the second set of measurements performed on the additional fabricated sample is in line with the first set of measurements with low differences.

\section{Conclusion}

The present study is dedicated to thermal measurements in conductive heat transfer tree-like structures obtained by topology optimization with an objective to analyse experimentally the thermal behaviour of the optimized structure under some specific thermal loads and boundary conditions. The optimal configuration obtained from TO was fabricated in aluminum-polymer combination and infrared thermography was used to measure the steady state temperature distribution on the optimal tree structure. CFD simulations were performed on the optimized structure to mimic exactly the same initial and boundary conditions that prevailed during the experiments whose results are then eventually compared to the experimental measurements. The optimized structures obtained by TO were experimentally found to be effective in reducing the overall average temperature in the domain. The values of objective function and maximum temperature measured by IR thermography and those obtained by CFD simulations were found to be in good agreement for the optimal structures under observation with $\varphi_{\max }=30 \%$. Finally, results of IR measurement reproduced on additional fabricated sample for the given optimal structure was found to be in line with the original IR thermal measurement thus ensuring reproducibility of experimental results. 


\section{References}

[1] M. P. Bendsøe, N. Kikuchi, "Generating optimal topologies in structural design using a homogenization method," Computer methods in applied mechanics and engineering, vol. 71, no. 2, pp. 197-224, 1988.

[2] A. Gersborg-Hansen, M. P. Bendsøe, \& O. Sigmund, "Topology optimization of heat conduction problems using the finite volume method," Structural and multidisciplinary optimization, vol. 31, no. 4, pp. 251-259, 2006.

[3] J. Dirker, J. P. Meyer, "Topology optimization for an internal heat-conduction cooling scheme in a square domain for high heat flux applications," Journal of Heat Transfer, vol. 135, no. 11, p. 111010, 2013.

[4] T. Dbouk, "A review about the engineering design of optimal heat transfer systems using topology optimization," Applied Thermal Engineering, vol. 112, pp. 841-854, 2017.

[5] G. Marck, M. Nemer, J. L. Harion, S. Russeil, D. Bougeard, "Topology optimization using the SIMP method for multiobjective conductive problems," Numerical Heat Transfer, Part B: Fundamentals, vol. 61, no. 6, pp. 439-470, 2012.

[6] M. P. Bendsoe, O. Sigmund, Topology optimization: theory, methods, and applications. Springer Science \& Business Media, 2013.

[7] T. E. Bruns, D. A. Tortorelli, "Topology optimization of non-linear elastic structures and compliant mechanisms," Computer Methods in Applied Mechanics and Engineering, vol. 190, no. 26, pp. 3443-3459, 2001.

[8] K. Svanberg, " The method of moving asymptotes - a new method for structural optimization," International journal for numerical methods in engineering, vol. 24, no. 2, pp. 359-373, 1987.

[9] T. Dbouk, J. L. Harion, "Performance of Optimization Algorithms Applied to Large Nonlinear Constrained Problems," American Journal of Algorithms and Computing, vol. 2, no. 1, pp. 32-56, 2015.

[10] A. Evgrafov, M. M. Gregersen, M. P. Sørensen, "Convergence of cell based finite volume discretizations for problems of control in the conduction coefficients," ESAIM: Mathematical Modelling and Numerical Analysis, vol. 45, no. 6, pp. 1059-1080, 2011. 